\title{
H2O2-responsive VEGF/NGF gene co-delivery nano- system achieves stable vascularization in ischemic hindlimbs
}

\section{Youlu Chen}

CAMS and PUMC: Chinese Academy of Medical Sciences \& Peking Union Medical College

\section{Zuoguan Chen}

CAMS PUMC: Chinese Academy of Medical Sciences and Peking Union Medical College

\section{Jianwei Duan}

CAMS and PUMC: Chinese Academy of Medical Sciences \& Peking Union Medical College

\section{Liang Gui}

CAMS PUMC: Chinese Academy of Medical Sciences and Peking Union Medical College

\section{Huiyang Li}

CAMS PUMC: Chinese Academy of Medical Sciences and Peking Union Medical College

\section{Xiaoyu Liang}

CAMS and PUMC: Chinese Academy of Medical Sciences \& Peking Union Medical College

Xinxin Tian

CAMS and PUMC: Chinese Academy of Medical Sciences \& Peking Union Medical College

Kaijing Liu

CAMS and PUMC: Chinese Academy of Medical Sciences \& Peking Union Medical College

\section{Yongjun Li}

CAMS PUMC: Chinese Academy of Medical Sciences and Peking Union Medical College Jing Yang ( $\square$ yangjing37@hotmail.com )

CAMS PUMC: Chinese Academy of Medical Sciences and Peking Union Medical College https://orcid.org/0000-0003-3848-5958

\section{Research}

Keywords: Vascular endothelial growth factor, Nerve growth factor, Gene therapy, Hydrogen peroxide, Angiogenesis

Posted Date: November 1st, 2021

DOI: https://doi.org/10.21203/rs.3.rs-994399/v1 
License: (c) (i) This work is licensed under a Creative Commons Attribution 4.0 International License. Read Full License

Version of Record: A version of this preprint was published at Journal of Nanobiotechnology on March 19th, 2022. See the published version at https://doi.org/10.1186/s12951-022-01328-6. 
$1 \mathrm{H}_{2} \mathrm{O}_{2}$-responsive $\mathrm{VEGF} / \mathrm{NGF}$ gene co-delivery nano-system achieves

2 stable vascularization in ischemic hindlimbs

3 Youlu Chen ${ }^{1, \uparrow}$, Zuoguan Chen ${ }^{2, \uparrow}$, Jianwei Duan ${ }^{1}$, Liang Gui ${ }^{2}$, Huiyang $\mathrm{Li}^{1}$, Xiaoyu

4 Liang ${ }^{1}$, Xinxin Tian ${ }^{1}$, Kaijing Liu ${ }^{1}$, Yongjun $\mathrm{Li}^{2}{ }^{2, *}$, Jing Yang ${ }^{1, *}$

51 Tianjin Key Laboratory of Biomaterial Research, Institute of Biomedical

6 Engineering, Chinese Academy of Medical Science and Peking Union Medical

7 College, 236 Baidi Road, Tianjin, 300192, P. R. China.

$8 \quad{ }^{2}$ Department of Vascular Surgery, Beijing Hospital, National Center of Gerontology,

9 Chinese Academy of Medical Science and Peking Union Medical College, 1 Dahua

10 Road, Beijing, 100730, P. R. China.

$11{ }^{*}$ Corresponding authors. E-mails: liyongjun4679@bjhmoh.cn;

12 yangjing37@hotmail.com;

$13{ }^{\dagger}$ These authors contributed equally to this work.

\section{Abstract}

15 Peripheral vascular disease (PVD) is a common clinical manifestation of 16 atherosclerosis. Vascular endothelial growth factor (VEGF) gene therapy is a 
17 promising approach for PVD treatment. However, due to single-gene therapy

18 limitations and high $\mathrm{H}_{2} \mathrm{O}_{2}$ pathological microenvironment, VEGF gene therapy are

19 not as expectations and its clinical application are limited. Synergistic effects of

20 Nerve factors and vascular factors in angiogenesis have attracted attention in recent

21 years. In this study, VEGF and nerve growth factor (NGF) genes co-delivery

22 nanoparticles (VEGF/NGF-NPs) were prepared by using $\mathrm{H}_{2} \mathrm{O}_{2}$ responsive

23 6s-PLGA-Po-PEG as a carrier. 6s-PLGA-Po-PEG could react with $\mathrm{H}_{2} \mathrm{O}_{2}$ specifically

24 due to the internal peroxalate bond. Angiogenic effects of VEGF/NGF-NPs has been

25 evaluated in cells and hindlimb ischemia mice model. Results showed that

26 VEGF/NGF-NPs promoted VEGF and NGF co-expression simultaneously, eliminated

27 excessive $\mathrm{H}_{2} \mathrm{O}_{2}$, strengthened reactions between SH-SY5Ys and HUVECs, and finally

28 enhanced migration, tube formation, proliferation and $\mathrm{H}_{2} \mathrm{O}_{2}$ damage resistance of

29 HUVECs. VEGF/NGF-NPs also recovered blood perfusion, promoted the expression

30 of VEGF, NGF, eNOS and NO, and enhanced vascular coverage of pericytes.

31 Treatment effects of VEGF/NGF-NPs may related to VEGF/eNOS/NO pathway.

32 Altogether, VEGF/NGF-NPs eliminated excessive $\mathrm{H}_{2} \mathrm{O}_{2}$ while achieving gene 
co-delivery, and promoted stable angiogenesis. It's a promising way for PVD treatment by using VEGF/NGF-NPs.

Keywords: Vascular endothelial growth factor, Nerve growth factor, Gene therapy, Hydrogen peroxide, Angiogenesis

\section{Introduction}

Peripheral vascular disease (PVD) is one of the most common vascular diseases, which refers to non-myocardial artery obstruction or stenosis, affecting more than 200 million people worldwide. PVD often showed lower extremity blood flow damage and increased hydrogen peroxide $\left(\mathrm{H}_{2} \mathrm{O}_{2}\right)$ content [1]. Blood vessel by-pass grafting and endovascular therapy have been regarded as "gold standard" among PVD treatments [2]. However, those surgeries require a long recovery time and may be associated with a variety of complications, such as graft infection, graft thrombosis, wound rupture or infection, and chronic lower extremity edema. In addition, there are many patients who don't meet the treatment conditions, and $30 \%$ of them will undergo extensive amputation [3]. These problems have brought great challenges to clinical treatment of PVD. 
Gene therapy is an attractive strategy to promote angiogenesis for repairing ischemic

50 tissue perfusion. It generates new blood vessels by transferring specific genes, such as

51 vascular endothelial growth factor gene (pVEGF) [4-5]. VEGF is a major regulator of

52 angiogenesis and a key factor in initiating complex cascades, which leads to the

53 formation of new vascular networks [6]. VEGF up-regulates nitric oxide synthase

54 (eNOS) protein level, and nitric oxide (NO) produced by eNOS significantly

55 contributes to the prosurvival/proangiogenic program of capillary endothelium by

56 triggering and transducing cell growth and differentiation [7-9]. However, clinical

57 trials of pVEGF therapy have been disappointing for poor stability of

58 neovascularization. ${ }^{[10]}$ This may be due to the limitations of single-gene therapy,

59 which is different from natural angiogenesis. Moreover, gene transfection efficiency

60 is poor in $\mathrm{H}_{2} \mathrm{O}_{2}$ microenvironment.

61 Angiogenesis is a complex process involving interconnections of molecular and tissue

62 signals, in which neural interaction is essential. It has been found that nerve growth

63 factor (NGF), as a pleiotropic factor acting on blood vessels and nerves, promotes

64 angiogenesis and vascular repair [10]. Therefore, co-delivery of pVEGF and NGF 
gene (pNGF) in ischemic hindlimb may expect to simulate interactive support of blood vessels and nerves, and enhance stable therapeutic angiogenesis ultimately.

Oxidative stress and redox regulation are also key issues in the PVD. Reactive oxygen species (ROS) is regarded as a double-edged sword in regulating various cellular signal transduction processes. When produced ROS exceed natural level of cells, it was found to cause destruction of nucleic acids, proteins, lipids and other biological molecules, and eventually trigger cell death [11]. Among numerous kinds of ROS, hydrogen peroxide $\left(\mathrm{H}_{2} \mathrm{O}_{2}\right)$ is the main component. Therefore, the use of $\mathrm{H}_{2} \mathrm{O}_{2}$ scavenging materials is expected to construct an immune microenvironment conductive angiogenesis. In our group, PLGA and PEG were covalently linked by peroxalate bonds, which could react with $\mathrm{H}_{2} \mathrm{O}_{2}$ specifically [12]. While reducing excess $\mathrm{H}_{2} \mathrm{O}_{2}$ in pathological microenvironment, NPs could realize the responsive release of genes, thus higher gene transfection efficiency could be achieved.

In this study, pVEGF and pNGF co-delivery nanoparticles (VEGF/NGF-NPs) were prepared by using 6s-PLGA-Po-PEG as carrier. The characterization of VEGF/NGF-NPs, including cellular uptake, lysosomal escape, genes transfection, 
$\mathrm{H}_{2} \mathrm{O}_{2}$ scavenging, cell migration, tube formation and intercellular interactions, were evaluated in vitro. Therapeutic effects of VEGF/NGF-NPs in angiogenesis were also evaluated in hindlimb ischemia mice model by detecting the expression of VEGF, $\mathrm{NGF}, \mathrm{CD} 31, \alpha \mathrm{SMA}, \mathrm{NG}_{2}$, eNOS, NO, the repair of muscle tissue and the recovery of blood perfusion in ischaemic hindlimbs.

\section{Materials and methods}

\subsection{Materials}

pVEGF and pNGF were purchased from Aibimeng Biotechnology Co., Ltd (Jiangsu, China). Protamine sulfate and coumarin 6 were obtained from Aladdin (Shanghai, China). Paraformaldehyde, DAPI, MTT, RIPA Lysis Buffer, $5 \times$ SDS-PAGE Sample Loading Buffer, $20 \times$ TBST buffer, ECL Western Blotting Substrate and Color Mixed Protein Marker were purchased from Solarbio (Beijing, China). SDS-PAGE gel preparation kit and Lyso-Tracker were bought from Beyotime (Jiangsu, China). Hydrogen peroxide $\left(\mathrm{H}_{2} \mathrm{O}_{2}, 30\right.$ wt \%) was purchased from Tianjin Guangfu Fine Chemical Research Institute (Tianjin, China). 2,7-dichlorodihydrofluorescein 
diacetate (DCFH-DA) was purchased from Sigma-Aldrich (St.Louis, MO, USA).

Matrigel was purchased from Corning (New York, USA).

\subsection{Cells and animals}

The human umbilical vein endothelial cells (HUVECs) and SH-SY5Ys cell line were obtained from the Cell Bank of Chinese Academy of Sciences. HBVPs were purchased from Shanghai Yubo Biotechnology Co., Ltd. Female 6-week-old ICR mice were purchased from SPF (Beijing) Biotechnology Co., Ltd. (China) (Approval No.: SCXK(Jing): 2019-0010).

\subsection{Preparation of NPs}

Preparation of VEGF-NPs (or NGF-NPs): distilled water solution containing $1.5 \mathrm{mg}$ protamine sulfate was slowly added into dichloromethane solution containing $80 \mathrm{mg}$ 6s-PLGA-Po-PEG under ice bath condition at $20000 \mathrm{rpm}$ of homogenizer. Under same conditions, distilled water solution containing $1.5 \mathrm{mg}$ of pVEGF (or pNGF) was added slowly into the above solution. Under the condition of No.3 Power of homogenizer, above solution was dropwise added into $15 \mathrm{~mL} 1 \%$ PVA solution slowly and stirred for $20 \mathrm{~min}$. Then, dichloromethane was removed by stirring and 
113 were obtained.

114 The preparation of Blank-NPs, Coumarin-NPs and VEGF/NGF-NPs: Blank-NPs were

115 prepared without any plasmids added; Coumarin-NPs were prepared with $8 \mathrm{mg}$ coumarin 6 added to replace plasmids; VEGF/NGF-NPs were prepared by adding 1.5 mg pVEGF, $1.5 \mathrm{mg} \mathrm{pNGF}$ and $3 \mathrm{mg}$ protamine sulfate. The remaining steps were the

118 same as above.

\subsection{Characterization of NPs}

120 Size, PDI and zeta potential of NPs were measured by zeta sizer nano ZS (Malvern

121 instruments, UK) ( $\mathrm{n}=3)$. Stability of NPs within $25 \mathrm{~d}$ was determined by zeta sizer

122 nano ZS every $5 \mathrm{~d}(\mathrm{n}=3)$.

123 Morphology of NPs: $100 \mu \mathrm{L}$ Blank-NPs, VEGF-NPs, NGF-NPs and VEGF/NGF-NPs

124 suspension were dropped on copper mesh. After drying overnight, the morphology of

125 NPs were observed by transmission electron microscopy (TEM) (h-6009iv, Hitachi). 
Gene's encapsulation: Supernatant was obtained by centrifuging NPs suspension, and

127 then agarose gel electrophoresis was used to detect whether there were unencapsulated genes in the supernatant.

Gene's release: After $2.8 \mathrm{mg}$ NPs were dissolved into $4 \mathrm{~mL}$ PBS (or $40 \mu \mathrm{M} \mathrm{H}_{2} \mathrm{O}_{2}$ solution), placed them in $37{ }^{\circ} \mathrm{C}$ constant temperature shaker. On the $1,3,5,8,12,16$, 20 and $25 \mathrm{~d}$, the above solutions were centrifuged at $15000 \mathrm{rpm}$ to get supernatants. After supernatants were collected the same volume of PBS was added. The content of plasmid was detected by nucleic acid densitometer at $260 \mathrm{~nm}$, and the cumulative

134 release curve was drawn $(n=3)$.

135 Cumulative release $(\%)=\frac{\text { Cumulative release of pDNA }}{\text { Total mass of loaded pDNA }} \times 100 \%$

\subsection{Celluar uptake and lysosomal escape of NPs}

HUVECs (or SH-SY5Ys) were seeded into confocal dish at a density of $5 \times 10^{4}$ cells/dish. HUVECs (or SH-SY5Ys) were incubated with $62.5 \mu \mathrm{g} / \mathrm{mL}$ coumarin

139 (naked-Coumarin group) or $687.5 \mu \mathrm{g} / \mathrm{mL}$ Coumarin-NPs (Coumarin-NPs group) for 0,

1402,4 and $6 \mathrm{~h}$ respectively. After cleaning with PBS for 3 times, add $400 \mu \mathrm{L}$

141 Lyso-Tracker Red to each dish, incubate for 4 h, and then clean with PBS for 3 times. 
142

After that, $500 \mu \mathrm{L} 4 \%$ paraformaldehyde was added to each dish for 20 min at RT and then PBS was used to gently cleaned for 3 times. Added $300 \mu \mathrm{L}$ DAPI dye to each dish and reacted at RT for 6 min, and then cleaned the dishes gently with PBS for 3 times. Finally, $500 \mu \mathrm{L}$ PBS was added to each dish. Celluar uptake and lysosomal escape of Coumarin-NPs in HUVECs (or SH-SY5Ys) were observed by confocal laser scanning microscopy (CLSM 410, Zeiss, Jena, Germany).

\subsection{Cytocompatibility and $\mathrm{H}_{2} \mathrm{O}_{2}$ scavenging ability}

Intracellular clearance of $\mathrm{H}_{2} \mathrm{O}_{2}$ : HUVECs (or SH-SY5Ys) were seeded into a confocal dish with a density of $5 \times 10^{4}$ cells/dish. After 24 h, HUVECs (or SH-SY5Ys) were incubated with $648.4 \mu \mathrm{g} / \mathrm{mL}$ Blank-NPs for $24 \mathrm{~h}$. Control group was added with the same volume of serum-free medium. After that, the medium containing $10 \mu \mathrm{g} / \mathrm{mL}$ LPS was replaced and incubated for $2 \mathrm{~h}$. The remaining $\mathrm{H}_{2} \mathrm{O}_{2}$ was determined with the amplex red assay. Finally, $500 \mu \mathrm{L}$ PBS was added to observe the scavenging effect of Blank-NPs on intracellular $\mathrm{H}_{2} \mathrm{O}_{2}$ by confocal laser scanning microscopy (CLSM 410, Zeiss, Jena, Germany). 
157

Response of NPs to $\mathrm{H}_{2} \mathrm{O}_{2}$ in vitro: After mixing $52.6 \mathrm{mg}$ Blank-NPs with $10 \mu \mathrm{M} \mathrm{H}_{2} \mathrm{O}_{2}$

medium (or PBS ) for $5 \mathrm{~min}$, the mixed liquid was dripped onto copper mesh. Dried overnight at RT, the morphological changes were observed under TEM (h-6009iv, Hitachi). The particle size, zeta potential of NPs were measured by zeta sizer nano ZS (Malvern instruments, UK).

Cytocompatibility of Blank- NPS on HUVECs and SH-SY5Ys : HUVECs (or SH-SY5Ys) were seeded into $96-$ well plates at a density of $5 \times 10^{3}$ cells/well. After 24 $\mathrm{h}$ of culture, HUVECs (or SH-SY5Ys) were incubated with 0.04, 0.17, 0.68, 2.70, 10.81, 43.23, $172.92 \mu \mathrm{g} / \mathrm{mL}$ Blank-NPs for $24 \mathrm{~h}$. Control group didn't add Blank-NPs. Then, $5 \mathrm{mg} / \mathrm{mL}$ MTT was added to each well. After $4 \mathrm{~h}$, supernatant was discarded and $150 \mu \mathrm{L}$ DMSO was added to each well. The cell plates vibrated for $5 \mathrm{~min}$ and the OD value of each well was detected at the wavelength of $490 \mathrm{~nm}$ by a Varioskan Flash microplate reader (Thermo Fisher Scientific, USA).

Cell viability $\%=\frac{\mathrm{OD} \text { value of Blank }-\mathrm{NPs} \text { group }}{\text { OD value of Control group }} \times 100 \%$

Protective effect of Blank-NPs on HUVECs in $\mathrm{H}_{2} \mathrm{O}_{2}$ solution: HUVECs were seeded into 96 -well plates at a density of $5 \times 10^{3}$ cells/well. After $24 \mathrm{~h}$ of culture, HUVECs 

medium. Then, $5 \mathrm{mg} / \mathrm{mL}$ MTT $20 \mu \mathrm{L}$ was added to each well. After $4 \mathrm{~h}$, supernatant was discarded and $150 \mu \mathrm{L}$ DMSO was added to each well. The cell plates vibrated for $5 \mathrm{~min}$ and the OD value of each well was detected at the wavelength of $490 \mathrm{~nm}$ by a Varioskan Flash microplate reader (Thermo Fisher Scientific, USA).

Cell viability $\%=\frac{\text { OD value of } \mathrm{H} 2 \mathrm{O} 2 \text { group }}{\mathrm{OD} \text { value of Control group }} \times 100 \%$

\subsection{Gene transfection of NPs}

HUVECs were seeded into confocal dishes at a density of $5 \times 10^{4}$ cells/well for $24 \mathrm{~h}$.

HUVECs were then incubated with $2.68 \mathrm{mg} / \mathrm{mL}$ NPs and $96.8 \mu \mathrm{g} / \mathrm{mL}$ naked $\mathrm{pVEGF}$ or/and pNGF respectively for $48 \mathrm{~h}$, and Control group was incubated with the same volume of serum-free medium. After 3 times of PBS cleaning, $500 \mu \mathrm{L} 4 \%$ paraformaldehyde was added to each dish at RT for $20 \mathrm{~min}$. After cleaning with PBS for 3 times, $300 \mu \mathrm{L}$ DAPI was added to each dish. After reacting at RT for $6 \mathrm{~min}$, 
gene transfection results by confocal laser scanning microscopy (CLSM 410, Zeiss, Jena, Germany).

\subsection{Cell migration and tube formation}

Cell migration: HUVECs (or HBVPs) were seeded into 6-well plates at a density of 3

$\times 10^{5}$ cells/well, and cultured for $24 \mathrm{~h}$ to achieve $100 \%$ fusion. Use pipette tip to make

scratch wound. Wash with PBS to remove the cells under scratch. HUVECs (or HBVPs) were incubated with $0.67 \mathrm{mg} / \mathrm{mL}$ NPs and $24.2 \mu \mathrm{g} / \mathrm{mL}$ naked pVEGF or/and pNGF respectively for $48 \mathrm{~h}$, and Control group was incubated with the same volume of serum-free medium. Cell migration was observed and photographed under inverted microscope at $12 \mathrm{~h}$ and $24 \mathrm{~h}$ respectively $(\mathrm{n}=4)$ and analysed by Image J software (National Institutes of Health, Bethesda, MD).

Cell migration $(\%)=\frac{\text { Initial scratch area-Scratch area after cell migration }}{\text { Initial scratch area }} \times 100 \%$ about $150 \mu \mathrm{L}$ of Matrigel was added into each 24 -well plate. The plate then placed into $37{ }^{\circ} \mathrm{C}$ incubator for $30 \mathrm{~min}$ to solidify the matrix. HUVECs was digested with 
or $24.2 \mu \mathrm{g} / \mathrm{mL}$ naked $\mathrm{pVEGF}$ or/and $\mathrm{pNGF}$ to prepare single cell suspension of $5 \times$ $10^{4}$ cells $/ \mathrm{mL}$. Control group cells were added with equal volume of serum-free medium. Then, HUVECs suspension was added into plate with matrix. After cultured in $37{ }^{\circ} \mathrm{C}$ incubator for $6 \mathrm{~h}$, tubule formation was observed under inverted microscope and photographed $(\mathrm{n}=4)$ and analysed by ImageJ software (National Institutes of Health, Bethesda, MD).

\subsection{Enhanced interaction between cells}

211 SH-SY5Ys (or HUVECs) were seeded into 6-well plates at a density of $3 \times 10^{5}$

212 cells/well, and cultured for $24 \mathrm{~h}$. SH-SY5Ys (or HUVECs) were then incubated with $2.68 \mathrm{mg} / \mathrm{mL}$ VEGF/NGF-NPs or Serum-free medium for $24 \mathrm{~h}$. After the incubation, supernatant was taken and centrifuged at $4000 \mathrm{rpm}$ for $10 \mathrm{~min}$ to get the SH-SY5Ys (or HUVECs) culture supernatant (Sup) and the SH-SY5Ys (or HUVECs) culture supernatant after VEGF/NGF-NPs incubation (Sup + NPs). On one hand, HUVECs

217 (or HBVPs) were incubated in serum-free medium or $10 \mu \mathrm{M} \mathrm{H}_{2} \mathrm{O}_{2}$ medium with Sup

218 or Sup + NPs for $24 \mathrm{~h}$, and Control group was incubated with the same volume of

219 serum-free medium to test cell proliferation ability followed the method in 2.6. On the 
other hand, HUVECs (or HBVPs) were incubated with Sup or Sup + NPs for $12 \mathrm{~h}$ and

$22124 \mathrm{~h}$, and Control group was incubated with the same volume of serum-free medium to test cell migration ability Followed the method in 2.8 .

\subsection{Blood perfusion and histological detection of hindlimbs}

Female ICR mice (6-week-old) were divided into 8 groups randomly: Control group

(PBS), VEGF group (naked VEGF), NGF group, VEGF + NGF group (naked NGF),

Blank-NPs group, VEGF-NPs group, NGF-NPs group and VEGF/NGF-NPs group.

Mice were anesthetized by intraperitoneal injection of chloral hydrate $(400 \mathrm{mg} / \mathrm{kg})$

and the femoral artery of mice was excised from inguinal ligament to bifurcation of saphenous artery and popliteal artery under general anesthesia. On the 1st, 3rd, 5th, were injected intramuscularly. PBS injection was used as control. On the 1st, 4th, 7th, 14th, 21st and 28th day after surgery, the blood perfusion of the hind limbs was monitored by Laser Doppler Flowmeter (PERIMED AB, Sweden) and results were analyzed by PIMSOFT software (PERIMED AB, Sweden) ( $n=5)$. After sacrifice of 
236 Masson staining. Angiogenesis was detected by CD31 immunohistochemistry and

237 vascular stability was detected by CD31, NG2, $\alpha$-SMA, DAPI immunofluorescence

238 staining.

\subsection{VEGF, NGF, eNOS and NO expression assay}

After homogenized in $400 \mu \mathrm{L}$ of lysis buffer, the lysates of ischaemic tissues were

242 collected from supernatants were electrophoresed on $10 \%$ polyacrylamide gels. Then,

243 proteins were transferred to PVDF membranes. After that, PVDF membranes were

244 incubated with VEGF, NGF and eNOS antibodies followed by a HRP-conjugated

245 anti-goat secondary antibody. Super Signal Ultra chemiluminescent reagent (Pierce,

246 Rockford, IL) was used to detecte protein immunoblot signals. The expression level

247 of the VEGF, NGF and eNOS protein was quantified with ImageJ software $(n=5)$. NO

248 was detected by NO ELISA Kit (Shanghai Lanpai Biotechnology Co., Ltd, China).

\subsection{Statistical analysis}


250 Statistical significance between multiple groups were performed with one-way

251 ANOVA. Data are expressed as the mean \pm standard deviation. Differences with a

252 value of $P<0.05$ were considered statistically significant.

\section{$253 \quad 3$ Results}

$254 \quad 3.1$ Preparation and characterization of NPs

255 Gene NPs were prepared by high speed stirring double emulsion method (Figure 1A),

256 GFP plasmid (pGFP) was used as a report gene. To increase the gene loading rate in

257 NPs, positively charged protamine was added, which could combine plasmids and

258 NPs by electrostatic interaction. As is shown in Figure 1C, we prepared protamine

259 loaded GFP-NPs (with pro group) and protamine free GFP-NPs (without pro group)

260 respectively. Meanwhile, GFP-NPs (with pro) were dissolved in dichloromethane and

261 were extracted to release the inside plasmids with distilled water (NP's extract group).

262 Agarose gel electrophoresis was used to detect NP's extracted solution and

263 supernatant after centrifugation of NPs. It could be seen that pGFP was fully packed

264 into NPs by adding protamine. In addition, the pGFP remained integral in the process

265 of preparation without fragmentation. 
In accordance with the foregoing method, Blank-NPs (without plasmids), VEGF-NPs

267 (containing $1.5 \mathrm{mg}$ pVEGF), NGF-NPs (containing $1.5 \mathrm{mg}$ pNGF) and

VEGF/NGF-NPs (containing $1.5 \mathrm{mg}$ pVEGF and $1.5 \mathrm{mg} \mathrm{pNGF}$ ) were prepared

respectively. Morphology of NPs were observed by TEM. It could be seen that NPs

were spherical with a layer of small spherical particles adsorbed on the surface. This

271 phenomenon may be caused by the formation of complex between protamine and

272 plasmid and adsorption on NPs surface. (Figure 1B). By detecting the supernatant,

273 there were no leaked genes (Figure 1D). Therefore, both pVEGF and pNGF were

274 encapsulated completely. Particle size, PDI and zeta potential of NPs were also

detected. Particle sizes of Blank-NPs, VEGF-NPs, NGF-NPs and VEGF/NGF-NPs

were $(258.4 \pm 7.6) \mathrm{nm},(244.9 \pm 13.6) \mathrm{nm},(231.8 \pm 6.5) \mathrm{nm}$ and $(205.2 \pm 1.9) \mathrm{nm}$,

277 respectively. PDI were $0.226 \pm 0.036,0.273 \pm 0.008,0.273 \pm 0.008$ and $0.217 \pm 0.008$,

278 and zeta potential were $-(2.70 \pm 0.16) \mathrm{mV},-(5.03 \pm 0.95) \mathrm{mV},-(6.70 \pm 0.49) \mathrm{mV}$

279 and $-(11.27 \pm 1.65) \mathrm{mV}$ respectively (Figure 1E). In addition, NPs were stable within 
Polymer-based gene delivery systems have been reported widely [13-15]. Gene delivery systems usually need to be more easily uptaken by cells, achieving lysosomal escape, releasing genes and scavenging $\mathrm{H}_{2} \mathrm{O}_{2}$ successfully in cells [16-18]. Therefore, $\mathrm{H}_{2} \mathrm{O}_{2}$ scavenge ability and gene delivery process of VEGF/NGF-NPs were explored. Firstly, morphology, particle size and zeta potential of NPs in $\mathrm{H}_{2} \mathrm{O}_{2}$ environment were observed to explore $\mathrm{H}_{2} \mathrm{O}_{2}$ responsiveness of NPs. Results showed that NPs was obviously broken in $10 \mu \mathrm{m} \mathrm{H}_{2} \mathrm{O}_{2}$ solution. Schematic diagram and representative images of TEM were shown (Figure 2A\&B). Particle size results showed that small particle fragments increased with time, proving that fragmentation of NPs was occurring continuously (Figure 2C). Zeta potential results showed that surface charge of Blank-NPs changed from a large peak to three small peaks, including the same peak as the original peak, the peak with large negative charge and the peak with

294 positive charge (Figure 2D). These two new peaks may be caused by the release of

295 protamine after NPs reacted with $\mathrm{H}_{2} \mathrm{O}_{2}$. In addition, gene's release was accelerated in 
$\mathrm{H}_{2} \mathrm{O}_{2}$ responsive and could be broken after reacting with $\mathrm{H}_{2} \mathrm{O}_{2}$.

Then, to explore gene delivery process of VEGF/NGF-NPs (Figure 3A), cytocompatibility, cellular uptake, lysosomal escape, intracellular $\mathrm{H}_{2} \mathrm{O}_{2}$ clearance, cell protection and gene transfection of NPs were explored. Cytocompatibility of NPs was tested in HUVECs and SH-SY5Ys, and results showed that NPs had almost no toxicity to both type of cells (Figure 2G\&H). Coumarin-NPs were prepared by loading coumarin 6 to observe the uptake of NPs by HUVECs. Compared with naked-Coumarin group, Coumarin-NPs could be absorbed more by HUVECs within the same amount of time (Figure 3B), and Coumarin 6-NPs also achieved lysosomal escape successfully (Figure $3 \mathrm{C}$ ). $\mathrm{H}_{2} \mathrm{O}_{2}$ scavenging and cell protection of NPs were

308 also detected in HUVECs. In Figure 3D, compared with Control group, Blank-NPs

309 attenuated ROS increasing successfully in HUVECs induced by LPS. After that,

310 protection of NPs on HUVECs in $\mathrm{H}_{2} \mathrm{O}_{2}$ medium was also tested. Compared with $\mathrm{H}_{2} \mathrm{O}_{2}$

311 group, Blank-NPs resisted $\mathrm{H}_{2} \mathrm{O}_{2}$ injury on HUVECs effectively, and the protection

312 was enhanced when NP's concentration increased (Figure 2H). Finally, gene 
314 plasmids and NGF-GFP plasmids with fluorescent protein were constructed by using PAAV-G-CMV-2A-RFP-blank and PAAV-G-CMV-2A-GFP-blank vectors. After co-delivery, transfecation efficiency of NPs was observed in confocal laser scanning microscopy. Compared with free pVEGF and pNGF, delivery through NPs achieved more efficient pVEGF and pNGF transfection. More importantly, VEGF/NGF-NPs realized simultaneous and efficient co-transfection of pVEGF and pNGF (Figure 3F).

\subsection{VEGF/NGF-NPs promoted cell migration and tubulogenesis of HUVECs}

321 Enhancement of endothelial cell migration and tubulogenesis is crucial to angiogenesis [19]. Therefore, we detected tubule formation and migration of HUVECs incubated by NPs, and representative images were given (Figure 4A\&D). promote tube formation or migration of HUVECs, while VEGF-NPs group and VEGF/NGF-NPs group promoted relative tube length, relative meshed area and HUVECs migration obviously. Compared with VEGF+NGF group, VEGF/NG-NPs 
329

gene delivery system enhanced function of its inside genes through efficient transfection. In addition, compared with VEGF-NPs group, VEGF/NGF-NPs group didn't promote the relative tube length, but promoted the relative meshed area visibly, implying that co-delivery of pNGF assisted pVEGF in accelerating vascular network formation of HUVECs (Figure 4B\&C). And compared with VEGF-NPs group, differences of HUVECs migration in VEGF/NGF-NPs group were little in $24 \mathrm{~h}$. Co-delivery of pNGF to help pVEGF on HUVECs migration was limited (Figure $4 \mathrm{E} \& \mathrm{~F})$.

\subsection{VEGF/NGF-NPs promoted intercellular interactions}

Nutrition of nerve plays an important role in the process of ischemic tissue repair. If interactions between nerve and blood vessel enhanced, it's hopeful to get further angiogenesis and ischemic repair [20-21]. Therefore, we investigated whether VEGF/NGF-NPs had the potential to promote interactions between nerve and blood vessel. In this study, SH-SY5Ys were incubated with VEGF/NGF-NPs and supernatant after centrifuging (Sup+NPs) were collected in vitro, and then HUVECs were incubated with Sup+NPs to simulate the interaction process (Figure 5A). 
Before that experiment, we first detected cellular uptake (Figure 5B), lysosomal escape (Figure 5C) and $\mathrm{H}_{2} \mathrm{O}_{2}$ clearance (Figure 5D) of NPs in SH-SY5Ys. It could be seen that NPs achieved lysosomal escape and higher cellular uptake in SH-SY5Ys. Meanwhile, NPs also removed excessive ROS in SH-SY5Ys caused by LPS (Figure 5D). Therefore, the gene delivery system was also feasible for SH-SY5Ys. Then, we examined the effects of Sup+NPs on migration, proliferation and $\mathrm{H}_{2} \mathrm{O}_{2}$ resistance of HUVECs. Firstly, we presented representative images of HUVECs migration results (Figure 5E). Compared with Control group, Sup+NPs group promoted cell migration significantly at $12 \mathrm{~h}$ and $24 \mathrm{~h}$, and the effects increased with time (Figure 5F\&G). Next results showed that HUVECs proliferation in Sup+NPs group was 2.05 times as much as that in Control group (Figure 5H). Even under the influence of $\mathrm{H}_{2} \mathrm{O}_{2}$, HUVECs proliferation in Sup+NPs group was 1.66 times as much as that in Control group (Figure 5I). Sup+NPs could still promote HUVECs proliferation under $\mathrm{H}_{2} \mathrm{O}_{2}$ injury. These results indicated that VEGF/ NGF-NPs enhanced interactions between SH-SY5Ys and HUVECs and further promoted migration, proliferation, $\mathrm{H}_{2} \mathrm{O}_{2}$ resistance of HUVECs markedly. 
361 Pericyte coverage is crucial to the stability of neovascularization, therefore,

362 interactions between HUVECs and HBVPs after VEGF/NGF-NPs transfection have

363 been also explored. HUVECs were incubated with VEGF/NGF-NPs, and centrifuged

364 supernatant (Sup+NPs) was collected in vitro, then HBVPs were incubated with

365 Sup+NPs to simulate interaction process (Figure 6A). Results showed that compared

366 with Control group, cell proliferation (Figure 6B) and migration (Figure 6C\&D) of

367 HBVPs in Sup+NPs group was significantly promoted, even in $\mathrm{H}_{2} \mathrm{O}_{2}$ environment.

368 These results suggested that VEGF/NGF-NPs also enhanced interactions between

369 HUVECs and HBVPs, and further promoted migration, proliferation and anti- $\mathrm{H}_{2} \mathrm{O}_{2}$

370 damage ability of HBVPs.

$371 \quad 3.5$ VEGF/NGF-NPs promoted stable angiogenesis in ischemic hindlimbs

372 Effects of VEGF/NGF-NPs on angiogenesis in vivo was detected. By establishing

373 ICR mouse model of ischaemic hindlimb, we used five-point intramuscular injection

374 of VEGF/NGF-NPs to promote angiogenesis (Figure 7A). Figure 7B showed

375 representative blood flow images after $28 \mathrm{~d}$ of ischemia. Compared to $21 \mathrm{~d}$, blood

376 flow decreased on $28 \mathrm{~d}$ in all groups except VEGF/NGF-NPs group, which may be 
group and VEGF-NPs group, blood flow of VEGF/NGF-NPs group recovered significantly on $28 \mathrm{~d}$ (Figure 5D). Then H\&E staining and Masson staining were performed on ischemic tissue. It could be seen that size and shape of muscle fibers in VEGF-NPs group and VEGF/NGF-NPs group were both uniform. Both VEGF-NPs and VEGF/NGF-NPs improved muscle injury and reduced the production of collagen fiber obviously (Figure 8A\&B).

To observe the neovascularization more intuitively, we detected CD31 expression in

385 ischemic tissues by immunohistochemistry, and representative pictures were given

(Figure 9A). Compared with Control group and VEGF-NPs group, VEGF/NGF-NPs group promoted the expression of CD31 significantly, indicating that VEGF/NGF-NPs promoted angiogenesis in ischemic tissue significantly. During this process, pNGF and pVEGF played a synergistic role. The coverage of smooth muscle

390 cells and pericytes often reflects the maturation and stability of neovascularization. To

391 explore neovascularization stability, we stained ischemic tissues by

392 immunofluorescence to co-located CD31, $\alpha$-SMA (marker of smooth muscle cells), 
DAPI and NG2 (marker of pericytes) of the vessels, and gave the representative picture (Figure 9B). The results showed that functional vessels $(10 \mu \mathrm{m}-70 \mu \mathrm{m})$ in Control group and Blank-NPs group were less. Pericytes coverage of VEGF group, NGF group, VEGF+NGF group, VEGF-NPs group and NGF-NPs group were insufficient (white arrow) while VEGF/NGF-NPs group had relatively complete pericytes coverage. These results suggested that pNGF and pVEGF promoted stable angiogenesis in a synergistic manner.

\subsection{VEGF/NGF-NPs enhanced the expression of VEGF, NGF, eNOS and NO}

401 Nitric oxide (NO) produced by endothelial cells through endothelial nitric oxide synthase (eNOS) is an important vasoactive compound [22-24]. NO is responsible for a variety of physiological and cellular processes, including endothelial cell migration, proliferation and angiogenesis. The function and activity of eNOS in endothelial cells are crucial to vascular integrity and homeostasis [25-26]. In addition, studies have shown that NGF could improve angiogenesis through VEGF/Akt/NO dependent mechanism [27]. Therefore, expressions of VEGF (Figure 10A), NGF (Figure 10B), 

expression of VEGF, NGF, eNOS and NO in ischaemic hindlimb compared with

411 Control group (4.16, 4.15, 6.17 and 4.13 times of Control group, respectively). It

412 should be noted that compared with VEGF-NPs group (or NGF-NPs group),

413 VEGF/NGF-NPs didn't promote further expression of VEGF (or NGF) in ischemic

414 lower limbs, but the expression of eNOS and NO was significantly promoted. This

415 result may suggest that synergistic effect of pNGF helped pVEGF promoting more

416 expressions of eNOS and NO.

\section{Discussion}

418 Angiogenesis is an important adaptive mechanism against ischemia [28-30].

419 Angiogenesis through gene therapy is undoubtedly a promising method for the treatment of PVD. pVEGF therapy has brought a brief promise to people, but has

421 failed to achieve satisfactory clinical results. Strong overexpression of VEGF by

422 different gene therapy vectors induces the growth of abnormal hemangiomatous vascular structures in skeletal muscle. Low expression of VEGF is safe, but not

424 sufficient to produce an effective therapeutic benefit. Appropriate VEGF angiogenic 
426 of angiogenic stimulation to have a real therapeutic effect on PVD. Therefore, it's

427 particularly important to promote stable angiogenesis.

428 After the budding of angiogenesis, original vascular plexus is remodeled extensively.

429 The remaining vessels are matured and stable, which marks the end of vascular

430 plasticity [31-32]. Vascular stability requires vascular maturation through recruitment

431 of parietal cells (like pericytes and smooth muscle cells) and establishment of

432 intercellular contact between parietal cells and the endothelium. In addition, pericytes

433 have long been considering to be the main regulator of vascular maturation and

434 stability, and the association with pericytes is necessary to make vessels independent

435 of sustained VEGF expression [33-35]. Among many tissues and signaling molecules

436 that influence the maturation and stability of new blood vessels, neural fiber network

437 is an important part that cannot be ignored. Vascular circulatory system and neural

438 fiber network have similar shape distribution and close ties, so strengthening the

439 interactions between them is conducive to stable angiogenesis. NGF was first isolated

440 from nervous tissue, it's one kind of pleiotropic factor that can act on both nerves and 
441 blood vessels. Studies have shown that NGF stimulation increased the expression of

442 Notch1 in a dose-dependent manner, which activated the Notch signaling pathway.

443 And Notch signaling pathway plays a key role in pericytes survival [36-39]. However,

444 it's unknown whether NGF promotes stable angiogenesis through its influence on

445 pericytes, and our study has answered this question. pNGF as the co-delivery gene of

446 pVEGF enhanced the recruitment of pericytes and strengthened the stability of

447 neovascularization in $28 \mathrm{~d}$. Notably, compared with pVEGF, co-delivery of pNGF in

448 ischemic hindlimbs promoted pericyte recruitment. In future studies, using gene

449 therapy to promote the recruitment and coverage of pericytes may be an effective way

450 to promote stable angiogenesis.

451 For stable angiogenesis, protective gene delivery vector for genes is equally important.

452 In this study, we synthesized 6s-PLGA-Po-PEG which eliminated $\mathrm{H}_{2} \mathrm{O}_{2}$. NPs based

on 6s-PLGA-Po-PEG protected genes from oxidative damage and improved the

454 microenvironment of cells by decreasing $\mathrm{H}_{2} \mathrm{O}_{2}$. In this study, the removal of $\mathrm{H}_{2} \mathrm{O}_{2}$

455 from the microenvironment by NPs was not complete, and it was still difficult to 
restore the lower limb steady-state microenvironment. In future, carrier with the

457 ability to regulate $\mathrm{H}_{2} \mathrm{O}_{2}$ in microenvironment should be explored.

458 Co-therapy of pVEGF with a second gene has been studied by many researchers to

459 promote stable angiogenesis. Through the co-delivery of pNGF and pVEGF, Bin Gao

et al. found that gene expressions of double genome group were significantly increased at mRNA and protein levels, which was at least 2 and 1.5 times of that in single genome group respectively. Moreover, they confirmed that co-delivery of two genes had a synergistic effect, which enhanced the proliferation, migration and angiogenesis of HUVECs significantly [40]. Aiki Marushima et al. achieved better hemodynamic recovery and ischemic protection through the co-delivery of VEGF and PDGF-BB, and increased collateral blood flow of chronic hypoperfusion [41]. Our study proved that, co-delivery of pVEGF and pNGF had better therapeutic efficiency on the stable angiogenesis of ischemic hindlimb. In numerous studies, co-therapy of pVEGF and a second gene had achieved "1+1>2" therapeutic effect. In the future, combination of polygene therapy and microenvironment improvement may enable 
471 gene NPs more functions, which is expected to become a promising way in PVD

472 treatment.

4735 Conclusion

474 In summary, we used $\mathrm{H}_{2} \mathrm{O}_{2}$ responsive 6s-PLGA-Po-PEG to co-loading pVEGF and

475 pNGF and prepared VEGF/NGF-NPs. VEGF/NGF-NPs eliminated $\mathrm{H}_{2} \mathrm{O}_{2}$ while

476 co-delivering pVEGF and pNGF, promoted stable neovascularization in ischemic

477 hindlimbs successfully by strengthening cell interactions between nerves and blood

478 vessels. Therefore, co-gene delivering NPs that simultaneously enhance neurovascular

479 interactions and improve $\mathrm{H}_{2} \mathrm{O}_{2}$ microenvironment may be a promising strategy to

480 achieve stable angiogenesis in PVD treatment.

481 Acknowledgments

482 You-Lu Chen and Zuo-Guan Chen contributed equally to this work. We acknowledge

483 financial support from the National Natural Science Foundation of China (82072080,

484 31771097, 81870351), CAMS Innovation Fund for Medical Sciences

485 (2017-12M-1-016, 2018-12M-1-002), Tianjin Research Program of Application

486 Foundation and Advanced Technology (17JCZDJC37400), National Key Research 
High-Tech Leader \& Teams of Tianjin Government and the Tianjin innovation

Promotion Plan Key Innovation Team of Immunoreactive Biomaterials.

\section{Author contributions}

492

Youlu Chen: Methodology, Validation, Formal analysis, Roles, Writing - original

draft. Zuoguan Chen: Methodology, Validation, Formal analysis, Roles, Writing -

original draft. Jianwei Duan: Investigation, Resources. Liang Gui: Investigation,

Resources. Huiyang Li: Formal analysis, Data curation. Xiaoyu Liang: Investigation,

Resources. Xinxin Tian: Investigation, Resources. Kaijing Liu: Formal analysis,

Data curation. Yongjun Li: Writing - review \& editing. Jing Yang:

Conceptualization, Writing - review \& editing, Project administration, Funding

499 acquisition.

\section{Availability of data and materials}

501 The data that support the findings of this study are available from the corresponding 
505 All animal experiments were carried out in accordance with the relevant guidelines

506 and regulations approved by the Center of Tianjin Animal Experiment ethics

507 committee and authority for animal protection. (License for use of experimental

508 animals: Approval No.: SYXK (Jin):2019-0002)

509 Consent for publication

510 All the authors agree with the publication.

511 Competing interest

512 The authors declare that they have no known competing financial interests or personal

513 relationships that could have appeared to influence the work reported in this paper.

\section{References}

515 1. E. Shabani Varaki, G.D. Gargiulo, S. Penkala, P.P. Breen, Peripheral vascular

516 disease assessment in the lower limb: a review of current and emerging non-invasive

517 diagnostic methods, Biomed. Eng. Online. 17(1) (2018) 61. 
2. Y. Xie, Y. Guan, S.H. Kim, M.W. King, The mechanical performance of weft-knitted/electrospun bilayer small diameter vascular prostheses, J. Mec.h Behav. Biomed. Mater. 61 (2016) 410-418.

3. G.V. Shrikhande, J.F. McKinsey, Use and abuse of atherectomy: where should it be used? Semin. Vasc. Surg. 21(4) (2008) 204-9. clinic and beyond, Gene. Ther. 19(6) (2012) 622-9. Expert. Rev. Cardiovasc. Ther. 8(8) (2010) 1127-38. R. Gianni-Barrera, Vascular endothelial growth factor biology for regenerative angiogenesis, Swiss. Med. Wkly. 149 (2019) w20011. endothelial nitric-oxide synthase expression via a KDR/Flk-1 receptor and a protein 

up-regulates nitric oxide synthase expression in endothelial cells, Cardiovasc. Res. 41(3) (1999) 773-80.

9. Y. Zhao, P.M. Vanhoutte, S.W. Leung, Vascular nitric oxide: Beyond eNOS, J. Pharmacol. Sci. 129(2) (2015) 83-94.

Rakugi, R. Morishita, Gene therapy in peripheral artery disease, Expert. Opin. Biol. Ther. 15(3) (2015) 381-90.

541 11. B. Nico, D. Mangieri, V. Benagiano, E. Crivellato, D. Ribatti, Nerve growth 542 factor as an angiogenic factor, Microvasc. Res. 75(2) (2008) 135-41.

544 effect of Bcl-xl overexpression against oxidative stress-induced vascular endothelial 545 cell injury and the role of the Akt/eNOS pathway, Int. J. Mol. Sci. 14(11) (2013) 
J. Yang, Hydrogen peroxide-responsive micelles self-assembled from a peroxalate ester-containing triblock copolymer, Biomater. Sci. 4(2) (2016) 255-7. 14. C.K. Chen, P.K. Huang, W.C. Law, C.H. Chu, N.T. Chen, L.W. Lo,

551 Biodegradable Polymers for Gene-Delivery Applications, Int. J. Nanomedicine. 15 (2020) 2131-2150.

15. M. Ramezani, M. Ebrahimian, M. Hashemi, Current Strategies in the Modification of PLGA-based Gene Delivery System, Curr. Med. Chem. 24(7) (2017) 728-739. 16. Q. Cai, L. Wang, G. Deng, J. Liu, Q. Chen, Z. Chen, Systemic delivery to central nervous system by engineered PLGA nanoparticles, Am. J. Transl. Res. 8(2) (2016)

17. C. Zylberberg, K. Gaskill, S. Pasley, S. Matosevic, Engineering liposomal nanoparticles for targeted gene therapy, Gene. Ther. 24(8) (2017) 441-452. 
19. J. Jo, Y. Tabata, How controlled release technology can aid gene delivery, Expert.

564 Opin. Drug. Deliv. 12(10) (2015) 1689-701.

565 20. A. Robinet, A. Fahem, J.H. Cauchard, E. Huet, L. Vincent, S. Lorimier, F.

566 Antonicelli, C. Soria, M. Crepin, W. Hornebeck, G. Bellon, Elastin-derived peptides

567 enhance angiogenesis by promoting endothelial cell migration and tubulogenesis

568 through upregulation of MT1-MMP, J. Cell. Sci. 118(Pt 2) (2005) 343-56.

56921 M. Guang, Y. Yao, L. Zhang, B. Huang, L. Ma, L. Xiang, J. Jin, P. Gong, The

570 effects of nerve growth factor on endothelial cells seeded on different titanium

571 surfaces, Int. J. Oral. Maxillofac. Surg. 44(12) (2015) 1506-13.

22. N. Hansen-Algenstaedt, P. Algenstaedt, C. Schaefer, A. Hamann, L. Wolfram, G.

Cingoz, N. Kilic, B. Schwarzloh, M. Schroeder, C. Joscheck, L. Wiesner, W. Ruther,

574 S. Ergun, Neural driven angiogenesis by overexpression of nerve growth factor,

23. D. Perez-Cremades, C. Bueno-Beti, J.L. Garcia-Gimenez, J.S. Ibanez-Cabellos, C. 
mediators release through a COX-NOS interaction in human endothelial cells, J. Cell.

Mol. Med. 21(8) (2017) 1584-1592.

24. C.A. Dias-Junior, S.B. Cau, J.E. Tanus-Santos, [Role of nitric oxide in the control of the pulmonary circulation: physiological, pathophysiological, and therapeutic implications], J. Bras. Pneumol. 34(6) (2008) 412-9.

25. J.E. Church, D. Fulton, Differences in eNOS activity because of subcellular localization are dictated by phosphorylation state rather than the local calcium environment, J. Biol. Chem. 281(3) (2006) 1477-88.

26. C.A. Meza, J.D. La Favor, D.H. Kim, R.C. Hickner, Endothelial Dysfunction: Is There a Hyperglycemia-Induced Imbalance of NOX and NOS? Int. J. Mol. Sci. 20(15) (2019).

27. U. Forstermann, W.C. Sessa, Nitric oxide synthases: regulation and function, Eur. Heart. J. 33(7) (2012) 829-37, 837a-837d.

28. C. Emanueli, M.B. Salis, A. Pinna, G. Graiani, L. Manni, P. Madeddu, Nerve growth factor promotes angiogenesis and arteriogenesis in ischemic hindlimbs, Circulation. 106(17) (2002) 2257-62. 
29. G. Lorier, C. Tourino, R.A. Kalil, Coronary angiogenesis as an endogenous response to myocardial ischemia in adults, Arq. Bras. Cardiol. 97(6) (2011) e140-8. 30. S. Yuan, C.G. Kevil, Nitric Oxide and Hydrogen Sulfide Regulation of Ischemic Vascular Remodeling, Microcirculation. 23(2) (2016) 134-45.

31. E. Toyota, T. Matsunaga, W.M. Chilian, Myocardial angiogenesis, Mol. Cell. Biochem. 264(1-2) (2004) 35-44. 32. P. Barbacena, J.R. Carvalho, C.A. Franco, Endothelial cell dynamics in vascular remodelling, Clin. Hemorheol. Microcirc. 64(4) (2016) 557-563. branching morphogenesis during blood vessel regeneration in zebrafish caudal fins, Dev. Biol. 264(1) (2003) 263-74.

34. D. von Tell, A. Armulik, C. Betsholtz, Pericytes and vascular stability, Exp. Cell. Yao, R.M. Zhou, S.J. Zhang, Q. Jiang, C. Zhao, B. Yan, Targeting 
610

611

612

613

614

615

616

617

618

619

620

621

622

623

624

625

diabetes-induced microvascular dysfunction, Proc. Natl. Acad. Sci. U. S. A. 116(15)

(2019) 7455-7464.

36. A.M. Figueiredo, P. Villacampa, R. Dieguez-Hurtado, J. Jose Lozano, P. Kobialka,

A.R. Cortazar, A. Martinez-Romero, A. Angulo-Urarte, C.A. Franco, M. Claret, A.M.

Aransay, R.H. Adams, A. Carracedo, M. Graupera, Phosphoinositide

3-Kinase-Regulated Pericyte Maturation Governs Vascular Remodeling, Circulation.

142(7) (2020) 688-704.

37. J.C. Park, I.B. Chang, J.H. Ahn, J.H. Kim, J.H. Song, S.M. Moon, Y.H. Park,

Nerve Growth Factor Stimulates Glioblastoma Proliferation through Notch1 Receptor

Signaling, J. Korean. Neurosurg. Soc. 61(4) (2018) 441-449.

38. K. Yamamizu, M. Iwasaki, H. Takakubo, T. Sakamoto, T. Ikuno, M. Miyoshi, T.

Kondo, Y. Nakao, M. Nakagawa, H. Inoue, J.K. Yamashita, In Vitro Modeling of

Blood-Brain Barrier with Human iPSC-Derived Endothelial Cells, Pericytes, Neurons, and Astrocytes via Notch Signaling, Stem. Cell. Reports. 8(3) (2017) 634-647.

39. T. Nadeem, W. Bogue, B. Bigit, H. Cuervo, Deficiency of Notch signaling in pericytes results in arteriovenous malformations, JCI. Insight. 5(21) (2020). 
627 single to a dual-gene delivery nanosystem: coordinated expression matters for 628 boosting the neovascularization in vivo, Biomater. Sci. 8(8) (2020) 2318-2328.

632 ischemia, J. Cereb. Blood. Flow. Metab. 40(2) (2020) 404-419.

A

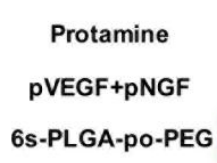

B

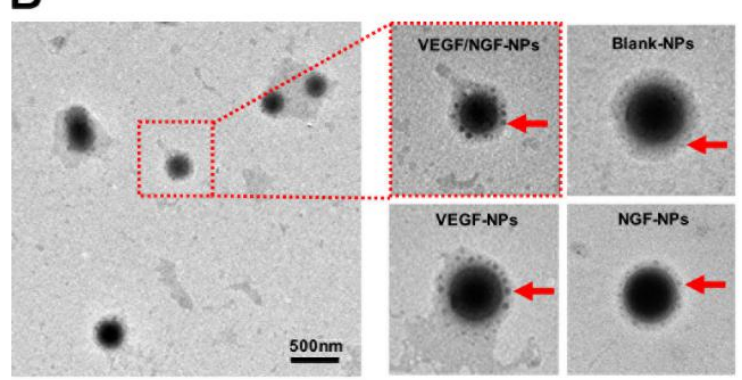

Double Emulsion

$\left(\mathbf{W}_{1} / O / \mathbf{W}_{2}\right)$

$$
\text { Solvent }
$$

Evaporation
C

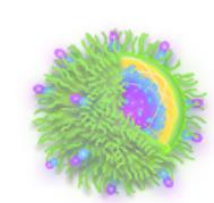

VEGF/NGF-NPS

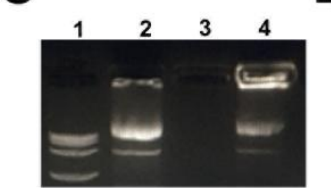

1: Marker

2: NPs without pro

3: NPs with pro

4: NP's extract

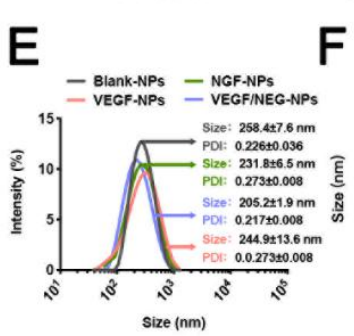

$F$
D

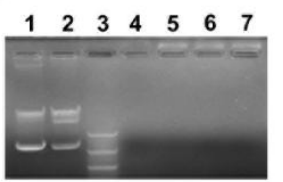

1: PVEGF 4: Blank-NPs 2: PNGF 5: VEGF-NPS 3: Marker 6: NGF-NPs 7:VEGF/NGF-NPS

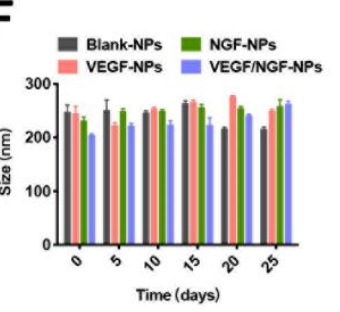

634

Figure 1. Characterization of NPs. (A) Schematic illustration of NP's preparation. (B) 
639 after protamine loaded NPs dissolving in dichloromethane. (D) Encapsulation 640 efficiency of gene NPs. (E) Particle size of NPs. (F) Stability of NPs within $25 \mathrm{~d}$. The 641 data are presented as mean $\pm \mathrm{SD}(\mathrm{n}=3)$.

A

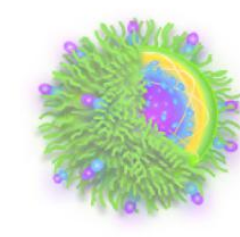

VEGF/NGF-NPs

B

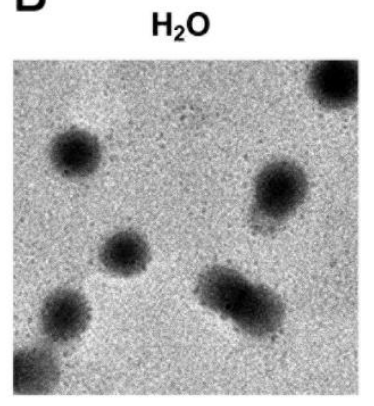

$\mathrm{H}_{2} \mathrm{O}_{2}$

\section{$\mathrm{H}_{2} \mathrm{O}_{2}$}

642

643

644

645

646

Figure $2 . \mathrm{H}_{2} \mathrm{O}_{2}$ responsiveness and biocompatibility of NPs
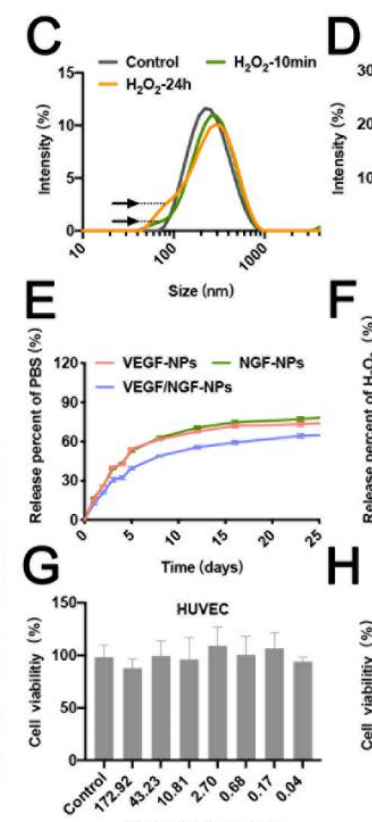

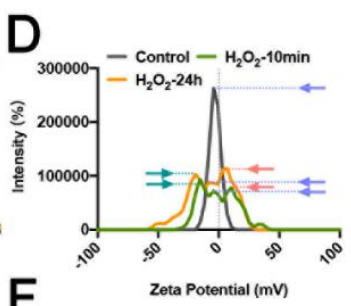

$F^{\frac{8}{8}}$
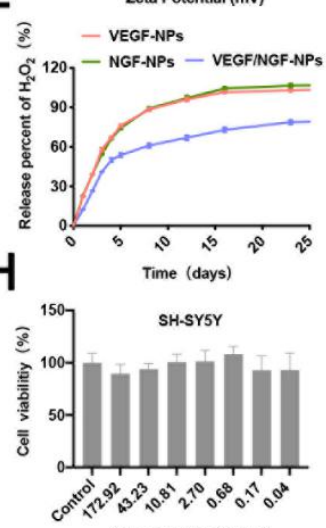

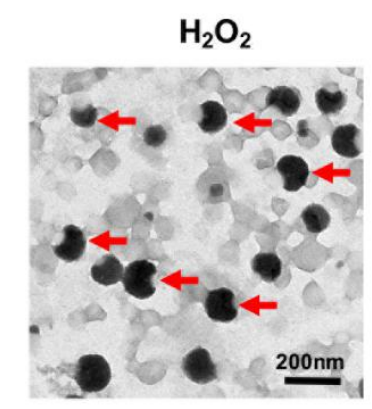

Concentration (ug/ml)

Concentration (ug/ml)

(A) Schematic illustration of NP's responsiveness to $\mathrm{H}_{2} \mathrm{O}_{2}$. (B) Typical images of NPs

in $\mathrm{H}_{2} \mathrm{O}$ or $\mathrm{H}_{2} \mathrm{O}_{2}$ under TEM. Particle size (C) and surface potential change (D) of Blank-NPs in PBS or $\mathrm{H}_{2} \mathrm{O}_{2}$. Genes release of NPs during $25 \mathrm{~d}$ in PBS (E) or $\mathrm{H}_{2} \mathrm{O}_{2}(\mathrm{~F})$. 

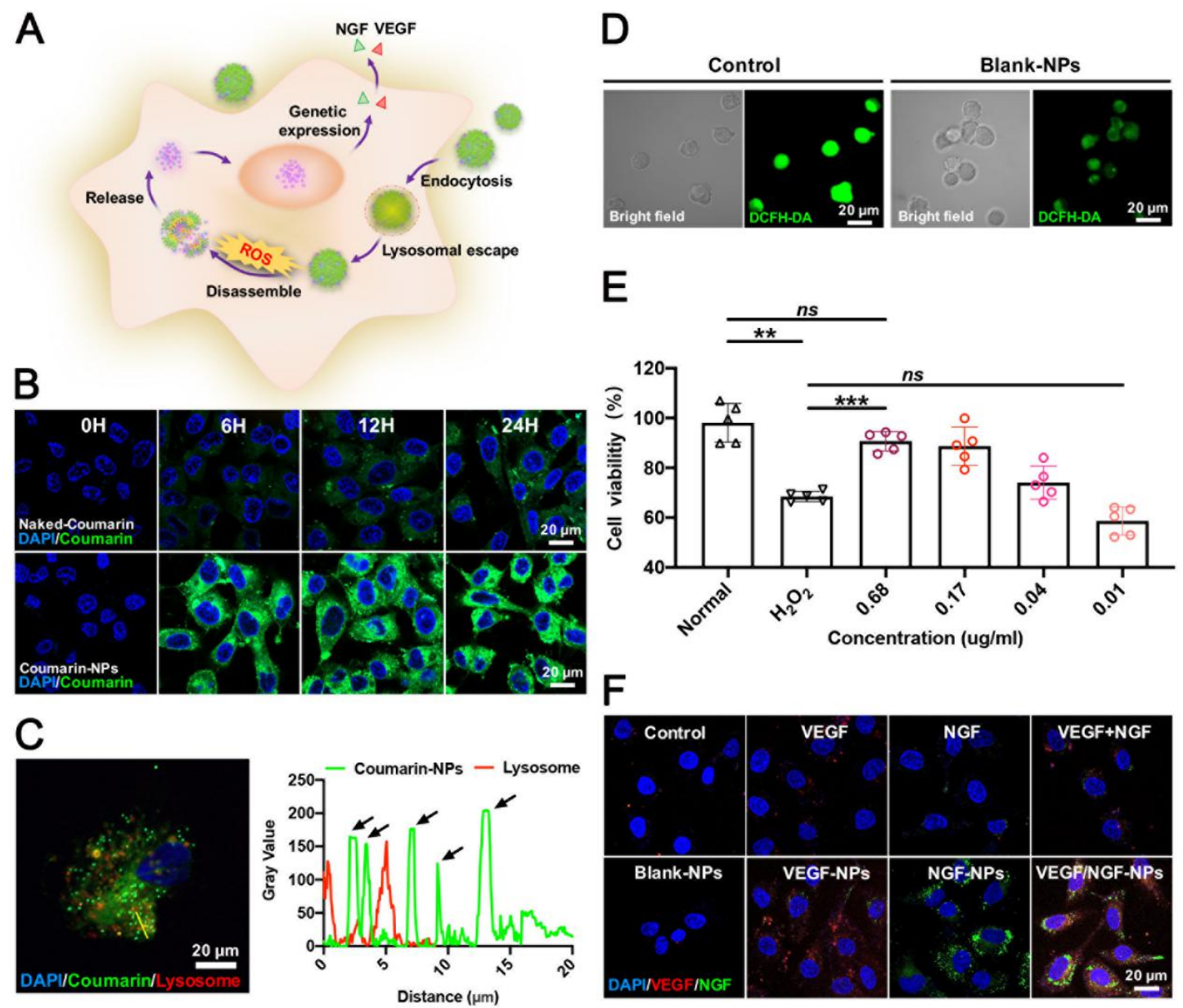

Figure 3. $\mathrm{H}_{2} \mathrm{O}_{2}$ scavenging and gene transfection of NPs in HUVECs. (A) Schematic

650 illustration of NP's ROS scavenging and pVEGF/pNGF co-transfection in cells. (B)

651 Cellular uptake of naked-Coumarin and Coumarin-NPs for $0 \mathrm{~h}, 6 \mathrm{~h}, 12 \mathrm{~h}$ and $24 \mathrm{~h}$. (C)

652 Lysosomal escape of Coumarin-NPs. (D) $\mathrm{H}_{2} \mathrm{O}_{2}$ scavenging of HUVECs after

653 incubating Blank-NPs $24 \mathrm{~h}$ in $10 \mu \mathrm{M}$ LPS. (E) Cell viability of HUVECs after

654 incubation with different concentrations of Blank-NPs in $10 \mu \mathrm{M} \mathrm{H}_{2} \mathrm{O}_{2}$. The data are

655 presented as mean $\pm \mathrm{SD}\left(\mathrm{n}=5,{ }^{* *} P<0.01,{ }^{* * *} P<0.001\right)$. (F) Gene co-transfection 656 through NPs. 
A
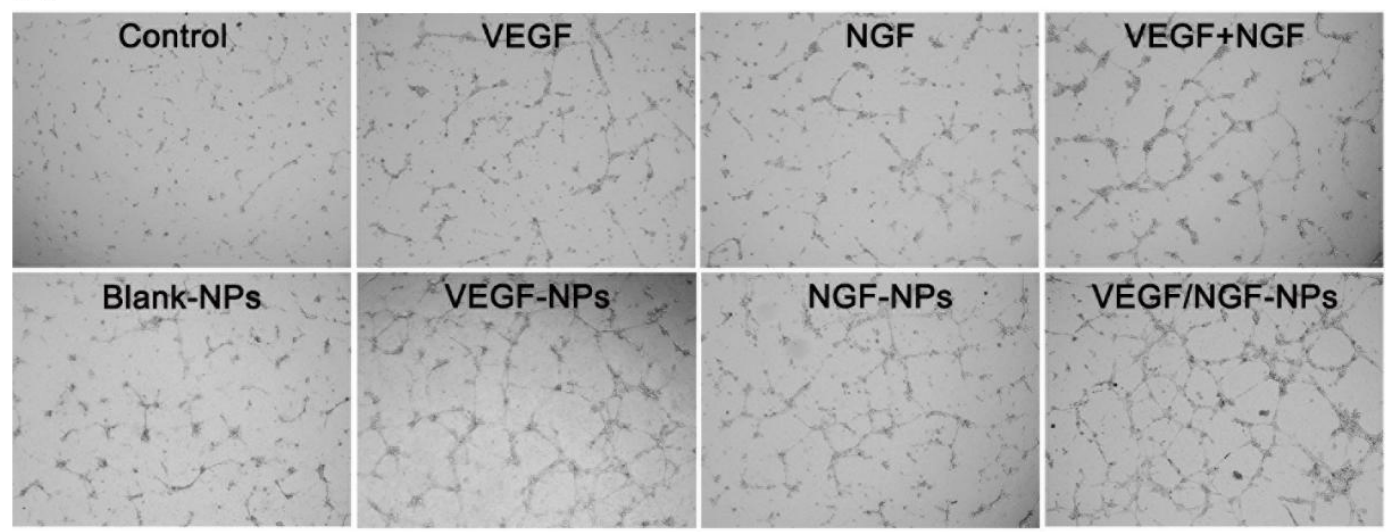

B

C
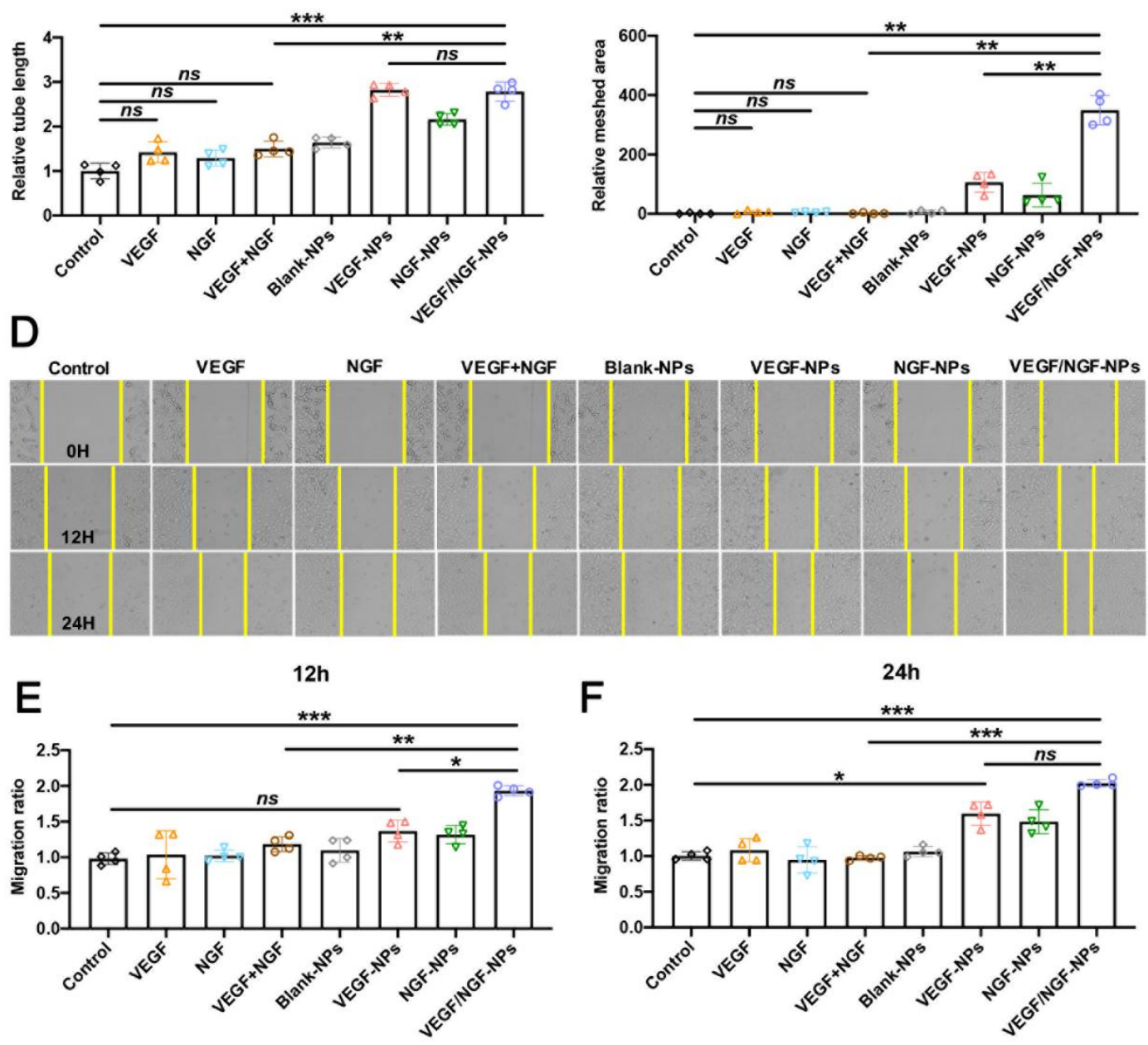

658 Figure 4. Effects of NPs on migration and tube fomation in HUVECs. (A) Images of

659 tubule formation after HUVECs incubated with NPs for $6 \mathrm{~h}$ was observed under

660 inverted microscop $(4 \times)$. Relative tube length (B) and relative meshed area(C) were 
661 also quantified. (D) Migration images of after HUVECs incubated with NPs for $12 \mathrm{~h}$

662 and $24 \mathrm{~h}$ was observed under inverted microscop $(4 \times)$. Quantification of cell

663 migration for $12 \mathrm{~h}(\mathrm{E})$ and $24 \mathrm{~h}(\mathrm{~F})$ were also quantified. The data are presented as

664 mean $\pm \mathrm{SD}\left(\mathrm{n}=4,{ }^{*} P<0.05,{ }^{* *} P<0.01,{ }^{* * *} P<0.001\right)$.
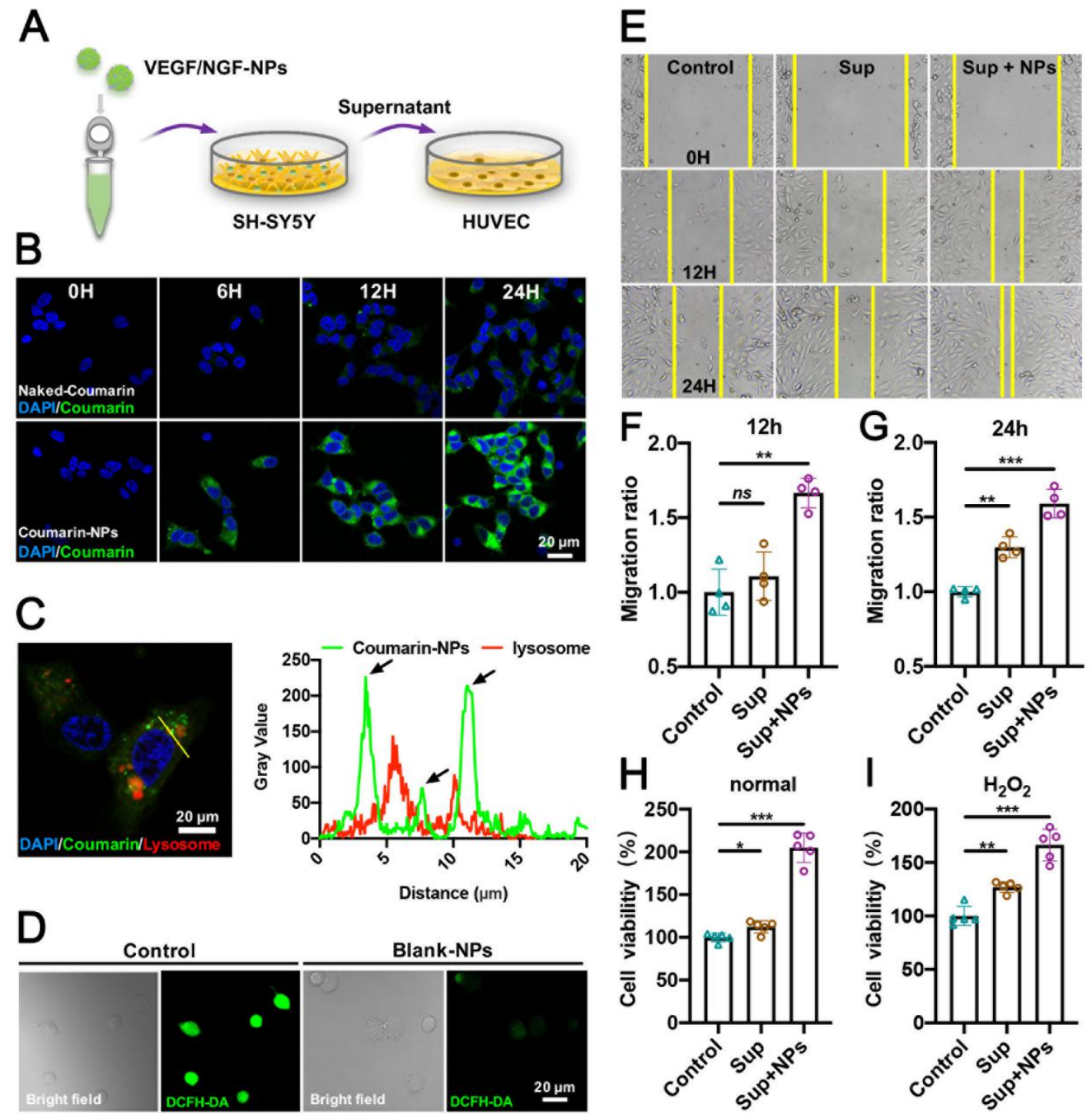

Figure 5. Enhanced interaction between SH-SY5Ys and HUVECs. (A) Schematic

667 illustration of interaction between SH-SY5Ys and HUVECs. After SH-SY5Ys was 

incubated with serum-free medium (Control), supernatant (Sup), 669 supernatant+VEGF/NGF-NPs (Sup+NPs) for $24 \mathrm{~h}$, HUVECs were incubated with 670 supernatant for $24 \mathrm{~h}$. (B) Cellular uptake of naked-Coumarin and Coumarin-NPs in 671 SH-SY5Ys for $0 \mathrm{~h}, 6 \mathrm{~h}, 12 \mathrm{~h}, 24 \mathrm{~h}$. (C) Lysosomal escape of Coumarin-NPs in 672 SH-SY5Ys. (D) H2O2 scavenging of SH-SY5Ys after incubating Blank-NPs in 10 $673 \mu \mathrm{M}$ LPS. (E) Migration images of HUVECs after incubating with serum-free medium, 674 Sup and Sup+NPs for $12 \mathrm{~h}$ and $24 \mathrm{~h}$. Quantification of cell migration for $12 \mathrm{~h}(\mathrm{~F})$ and $67524 \mathrm{~h}(\mathrm{G})$ were also quantified and the data are presented as mean $\pm \mathrm{SD}(\mathrm{n}=4, * * \mathrm{P}<$ $6760.01, * * * \mathrm{P}<0.001)$. Cell viabilities of HUVECs after incubating with serum-free 677 medium, Sup and Sup+NPs in PBS (H) and $10 \mu \mathrm{M} \mathrm{H}_{2} \mathrm{O}_{2}(\mathrm{I})$ and the data are presented 678 as mean $\pm \mathrm{SD}(\mathrm{n}=5, * * P<0.01, * * * P<0.001)$. 
A

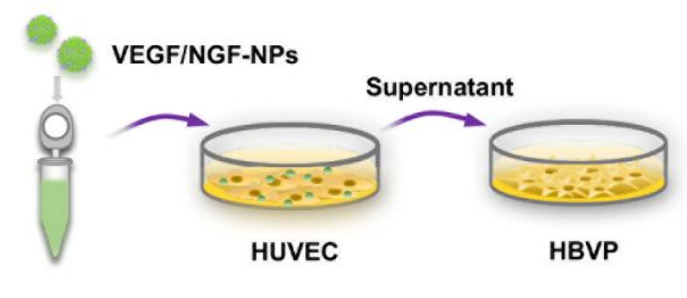

C

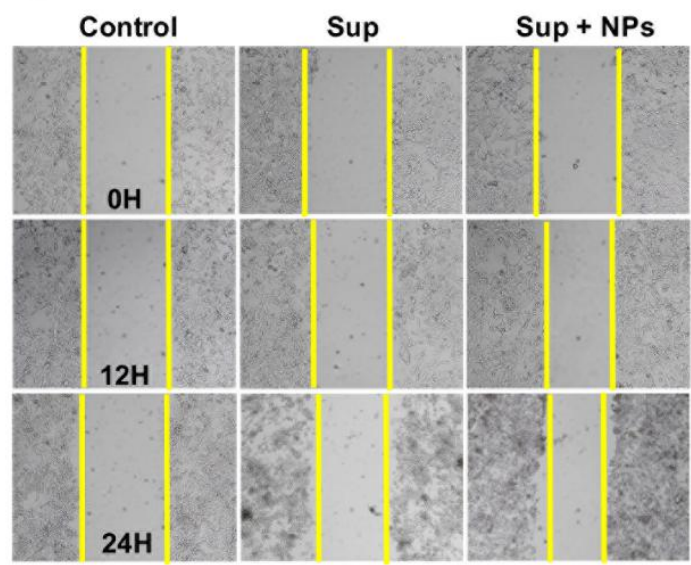

B
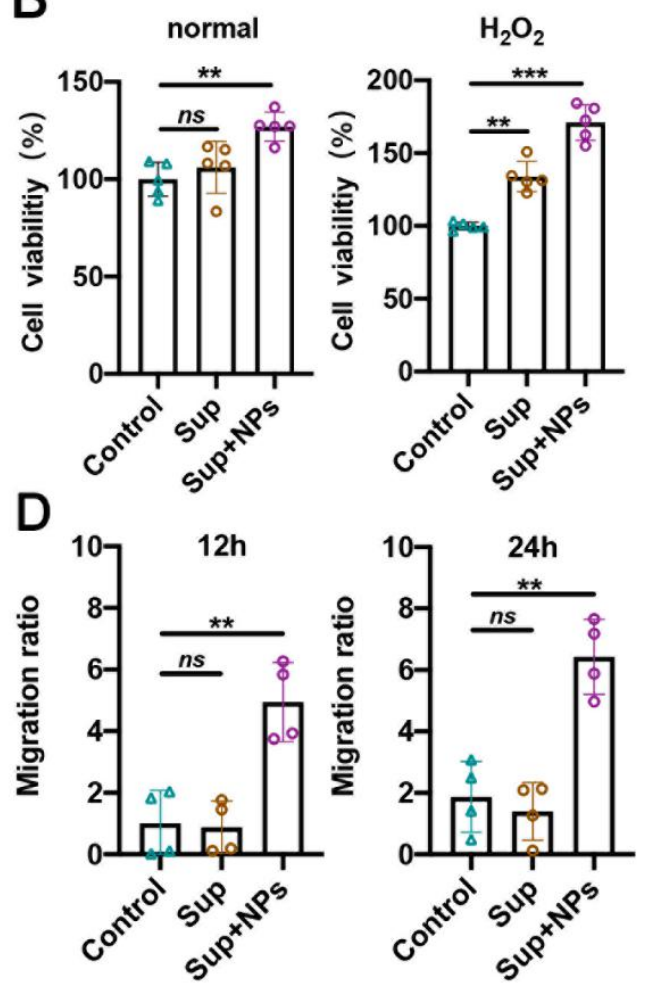

679

680 Figure 6. Enhanced interaction between HUVECs and HBVPs.

681 (A) Schematic illustration of interaction between HUVECs and HBVPs. (B) Cell

682 viabilities of HUVECs after incubating with serum-free medium, Sup and Sup+NPs

683 in $\mathrm{PBS}$ and $\mathrm{H}_{2} \mathrm{O}_{2}$ and the data are presented as mean $\pm \mathrm{SD}(\mathrm{n}=5, * * P<0.01, * * * P<$

684 0.001). (C) Migration images of HBVPs after incubating with serum-free medium,

685 Sup and Sup+NPs for $12 \mathrm{~h}$ and $24 \mathrm{~h}$. Quantification of cell migration for $12 \mathrm{~h}(\mathrm{~F})$ and 686 $24 \mathrm{~h}(\mathrm{D})$. 


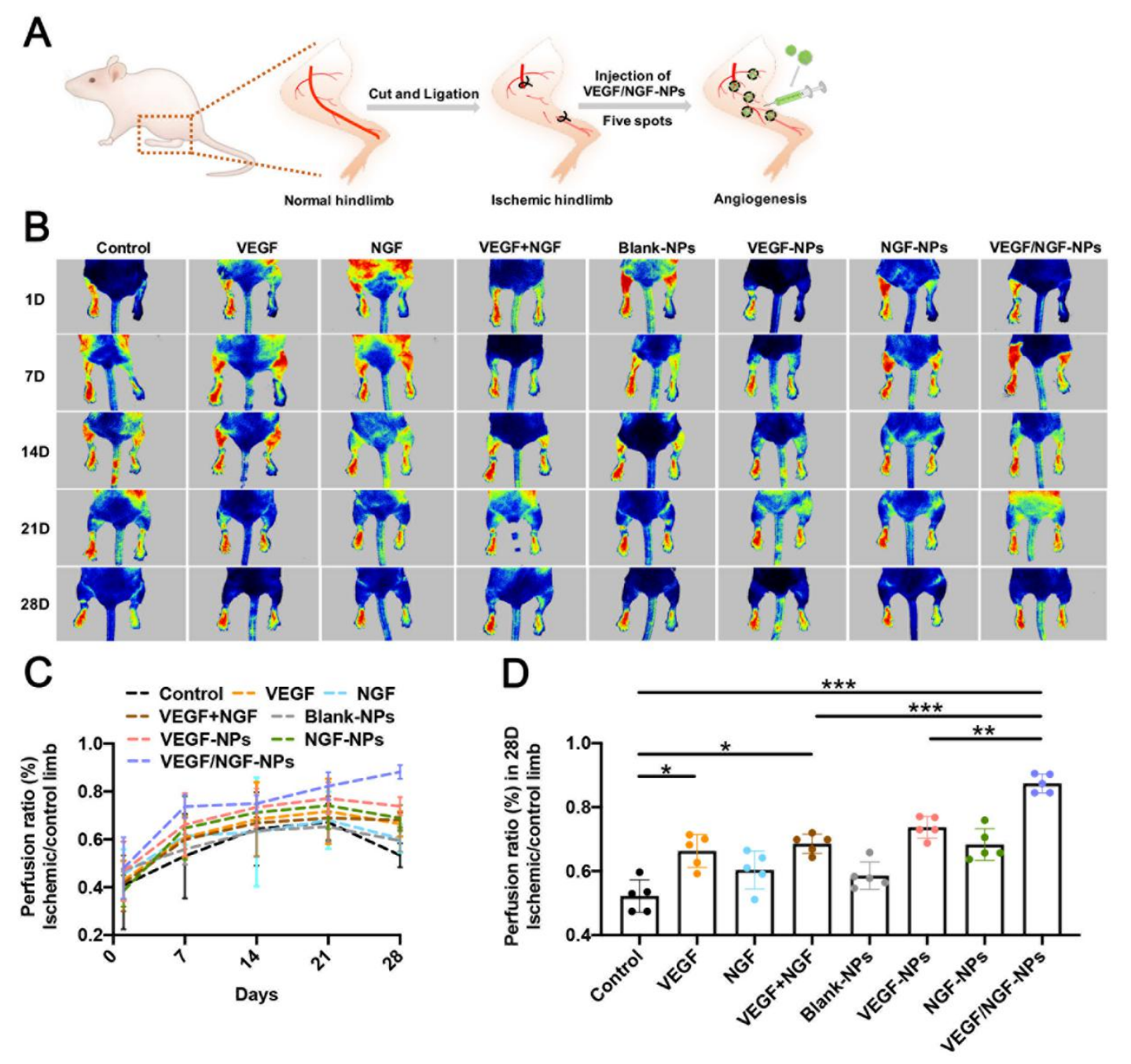

688 Figure 7. NPs affected blood reperfusion of the ischemic hindlimbs. (A) Schematic

689 diagram of ischaemic hindlimb model construction and VEGF/NGF-NPs promoting

690 angiogenesis. (B) Intramuscular injection of NPs to target blood flow recovery in

691 ischaemic hindlimb was investigated on $28 \mathrm{~d}$ and representative laser doppler

692 perfusion images were presented $(n=5)$. And statistical difference on $28 \mathrm{~d}$ were also

693 presented. The data are presented as mean $\pm \mathrm{SD}\left(\mathrm{n}=5,{ }^{*} P<0.05,{ }^{* *} P<0.01,{ }^{* * *} P<\right.$ 


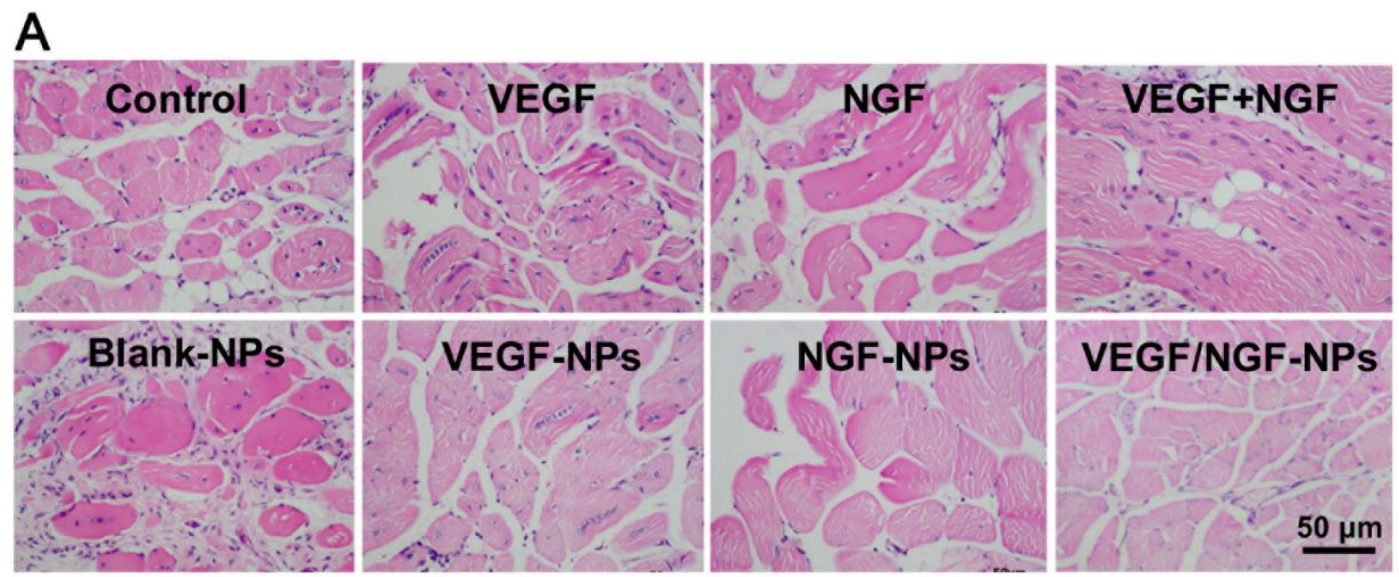

B

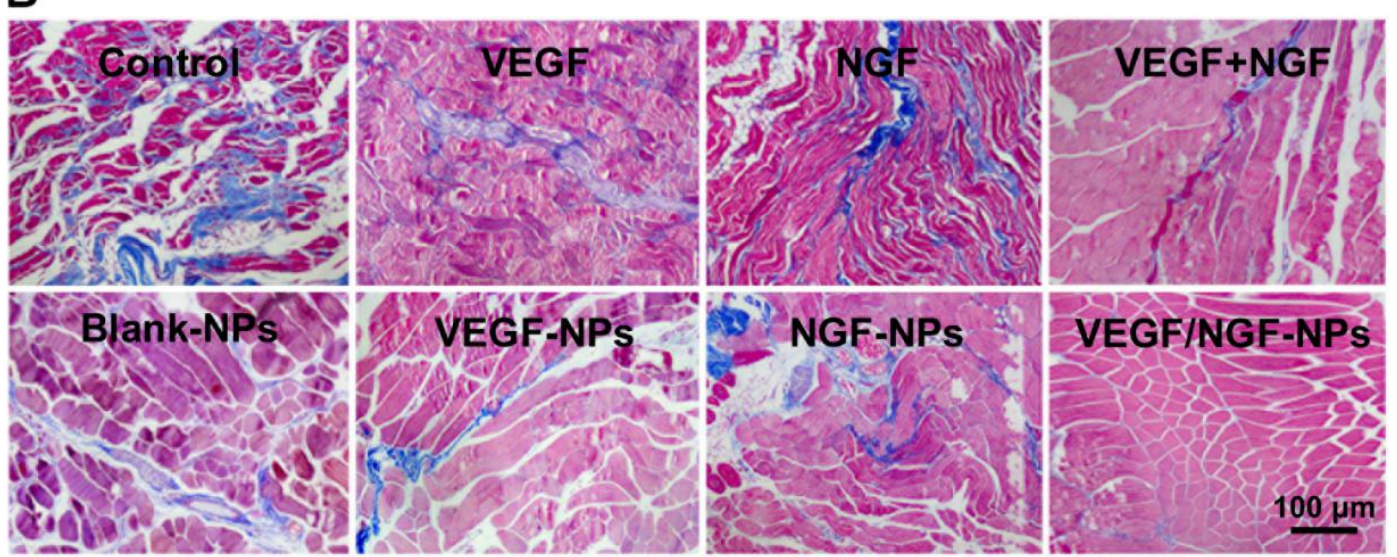

696 Figure 8. Histological evaluation of ischaemic hindlimb after treated by NPs.

697 Haematoxylin and Eosin (H\&E) staining (A) and Masson staining (B) of ischaemic

698 hindlimb tissue treated by NPs were presented. 
A
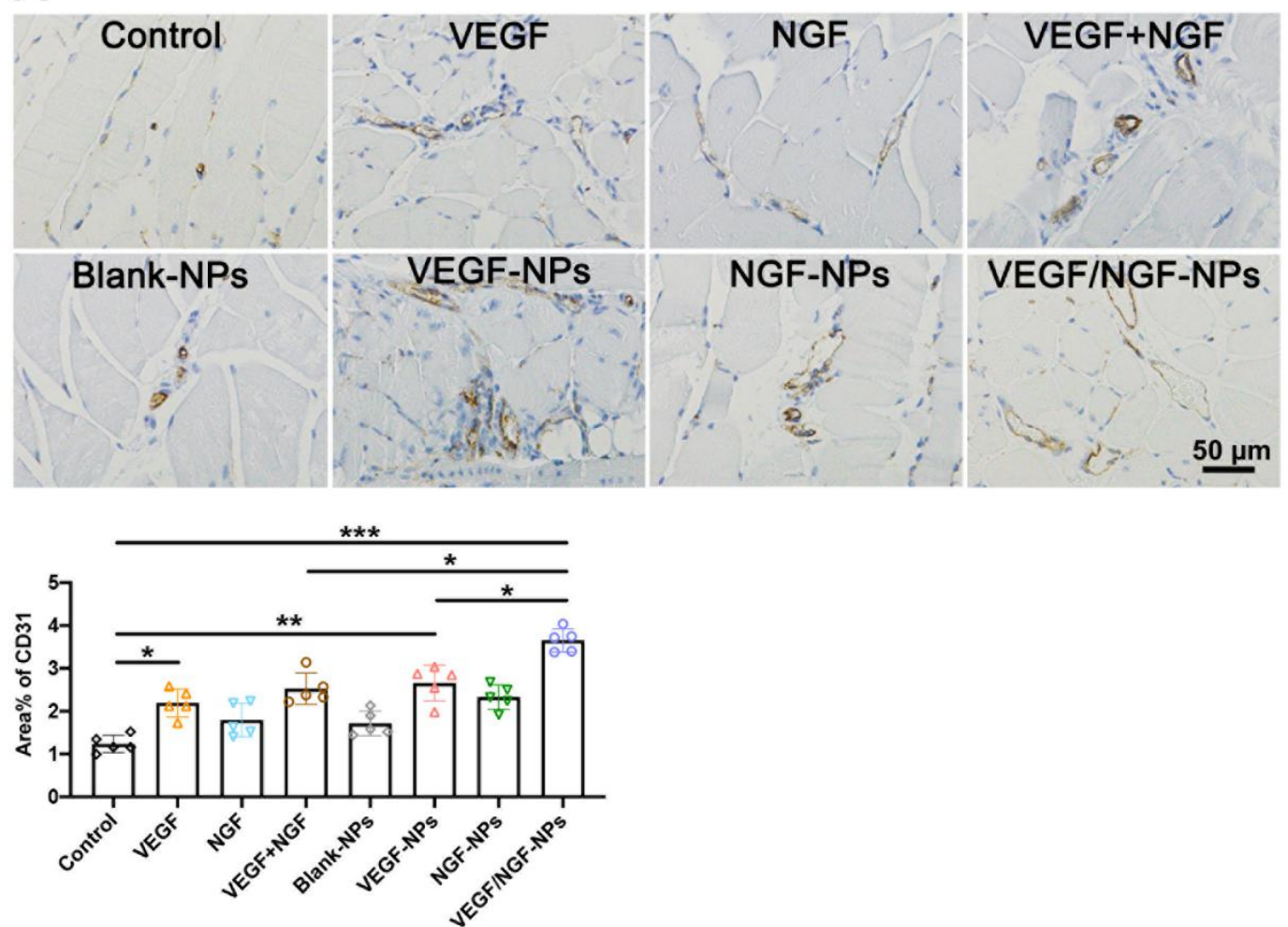

B

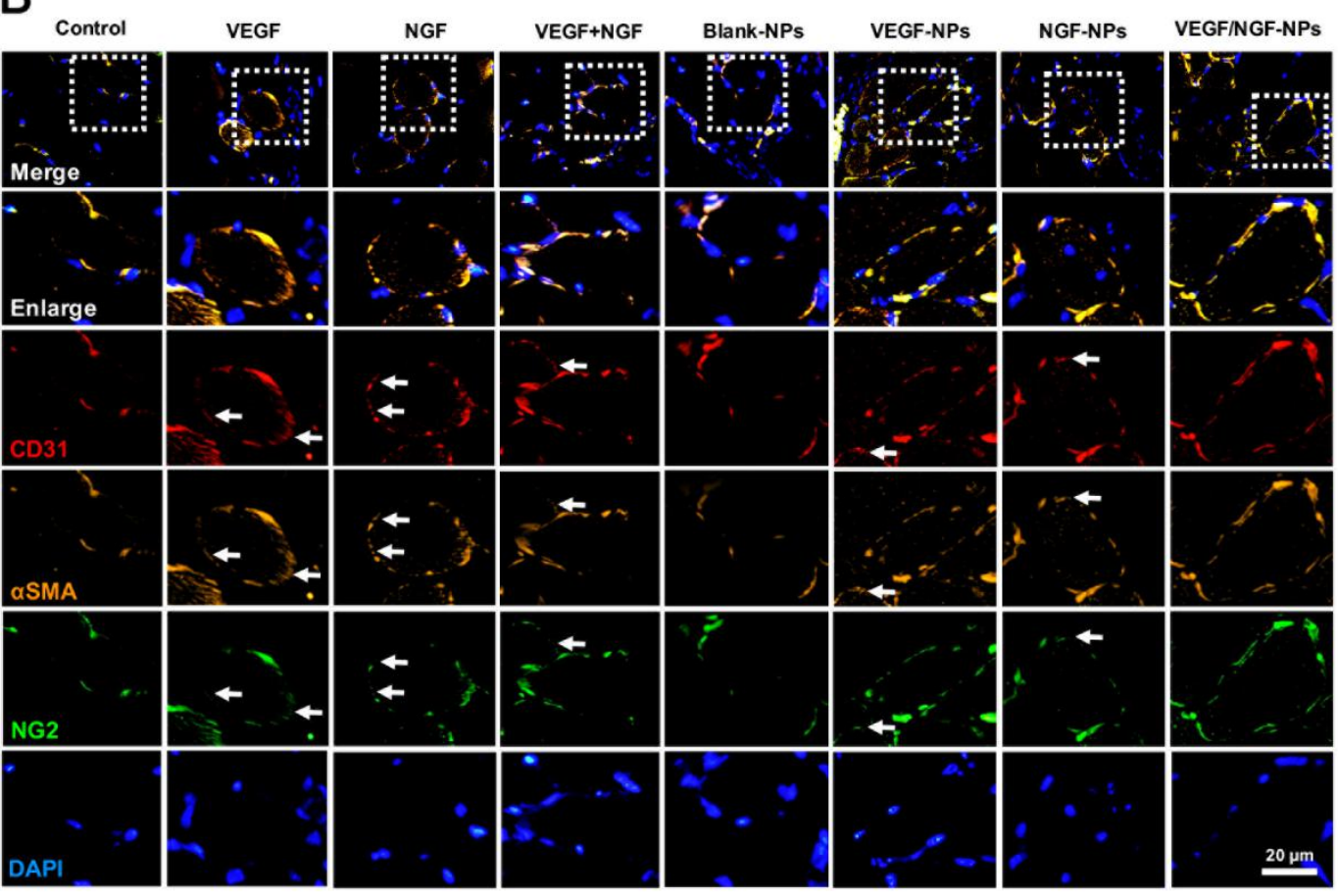

700 Figure 9. Angiogenesis and vascular stability of ischaemic hindlimb after treated by

701 NPs. (A) Quantification of CD31-positive areas. The data are presented as mean $\pm \mathrm{SD}$ 

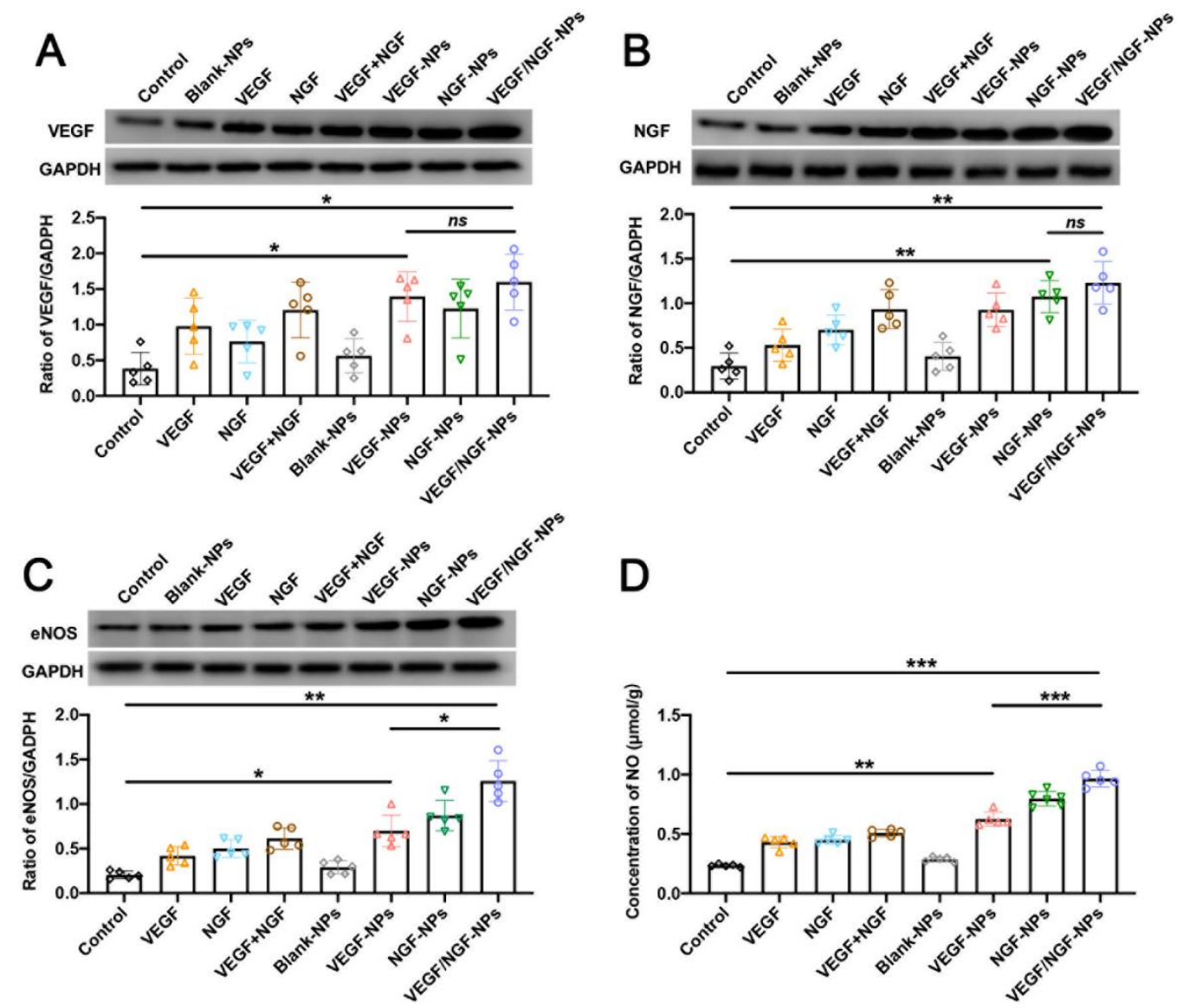

D

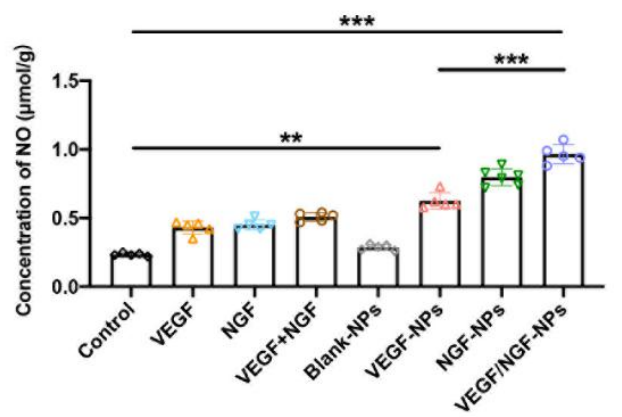

704

705 Figure 10. VEGF, NGF, eNOS and NO expression of ischaemic hindlimb after treated by NPs. Protein level alteration of VEGF (A), NGF (B), eNOS (C) was verified by western blot and NO (D) level alteration was verified by ELISA. All the data are presented as mean $\pm \mathrm{SD}(\mathrm{n}=5, * P<0.05, * * P<0.01, * * * P<0.001)$. 


\section{Supplementary Files}

This is a list of supplementary files associated with this preprint. Click to download.

- GraphicalAbstract.docx 